TRANSACTIONS OF THE

AMERICAN MATHEMATICAL SOCIETY

Volume 362, Number 11, November 2010, Pages 5967-5982

S 0002-9947(2010)05026-3

Article electronically published on June 16, 2010

\title{
ON THE LAW OF THE ITERATED LOGARITHM FOR THE DISCREPANCY OF LACUNARY SEQUENCES
}

\author{
CHRISTOPH AISTLEITNER
}

\begin{abstract}
A classical result of Philipp (1975) states that for any sequence $\left(n_{k}\right)_{k \geq 1}$ of integers satisfying the Hadamard gap condition $n_{k+1} / n_{k} \geq q>$ $1(k=1,2, \ldots)$, the discrepancy $D_{N}$ of the sequence $\left(n_{k} x\right)_{k \geq 1} \bmod 1$ satisfies the law of the iterated logarithm (LIL), i.e.

$$
1 / 4 \leq \limsup _{N \rightarrow \infty} N D_{N}\left(n_{k} x\right)(N \log \log N)^{-1 / 2} \leq C_{q} \quad \text { a.e. }
$$

The value of the lim sup is a long-standing open problem. Recently Fukuyama explicitly calculated the value of the $\lim \sup$ for $n_{k}=\theta^{k}, \theta>1$, not necessarily integer. We extend Fukuyama's result to a large class of integer sequences $\left(n_{k}\right)$ characterized in terms of the number of solutions of a certain class of Diophantine equations and show that the value of the limsup is the same as in the Chung-Smirnov LIL for i.i.d. random variables.
\end{abstract}

\section{INTRODUCTION}

Given a sequence $\left(x_{1}, \ldots, x_{N}\right)$ of real numbers, the value

$$
D_{N}=D_{N}\left(x_{1}, \ldots, x_{N}\right)=\sup _{0 \leq a<b \leq 1}\left|\frac{A(N, a, b)}{N}-(b-a)\right|
$$

is called the discrepancy of the sequence $\bmod 1$. Here $A(N, a, b)$ denotes the number of indices $k \leq N$ with $a \leq\left\langle x_{k}\right\rangle\langle b$, and $\langle\cdot\rangle$ denotes fractional part. An infinite sequence $\left(x_{n}\right)_{n \geq 1}$ is called uniformly distributed $\bmod 1$ if $D_{N}\left(x_{1}, \ldots, x_{N}\right) \rightarrow 0$ as $N \rightarrow \infty$. By a classical result of $\mathrm{H}$. Weyl [20, for any increasing sequence $\left(n_{k}\right)_{k \geq 1}$ of integers, the sequence $\left\langle n_{k} x\right\rangle$ is uniformly distributed for almost all real $x$ in the sense of Lebesgue measure. There is an extensive literature dealing with the asymptotic properties of the sequence $\left\langle n_{k} x\right\rangle$ (see Gaposhkin [10] for a survey until 1966), but the precise order of magnitude of its discrepancy has been found only for a few special sequences $\left(n_{k}\right)$. R.C. Baker 2 proved, improving earlier results of Erdős and Koksma [8] and Cassels [7, that for any increasing sequence $\left(n_{k}\right)$ of integers the discrepancy of $\left\langle n_{k} x\right\rangle$ satisfies

$$
D_{N}\left(n_{k} x\right)=O\left(N^{-\frac{1}{2}}(\log N)^{\frac{3}{2}+\varepsilon}\right) \quad \text { a.e. }
$$

Received by the editors June 2, 2008 and, in revised form, February 19, 2009.

2000 Mathematics Subject Classification. Primary 11K38, 42A55, 60F15.

Key words and phrases. Discrepancy, lacunary series, law of the iterated logarithm.

This research was supported by the Austrian Research Foundation (FWF), Project S9603-N13.

(C)2010 American Mathematical Society Reverts to public domain 28 years from publication 5967 
for every $\varepsilon>0$. (For simplicity, here and in the sequel we write $D_{N}\left(n_{k} x\right)$ instead of $D_{N}\left(n_{1} x, \ldots, n_{N} x\right)$.) In the case $n_{k}=k$, Kesten [14] proved

$$
D_{N}\left(n_{k} x\right) \sim \frac{2}{\pi^{2}} \frac{\log N \log \log N}{N} \quad \text { in measure. }
$$

The proof depends on the classical connection of the discrepancy of $\langle k x\rangle$ with the continued fraction expansion of $x$ and uses deep probabilistic ideas. Philipp 16 proved that if the sequence $\left(n_{k}\right)$ satisfies the Hadamard gap condition

$$
n_{k+1} / n_{k}>q>1, \quad k=1,2, \ldots,
$$

then the discrepancy of $\left\langle n_{k} x\right\rangle$ obeys the law of the iterated logarithm, i.e.

$$
\frac{1}{4 \sqrt{2}} \leq \limsup _{N \rightarrow \infty} \frac{N D_{N}\left(n_{k} x\right)}{\sqrt{2 N \log \log N}} \leq C_{q} \quad \text { a.e. }
$$

where $C_{q}$ is a constant depending on $q$. This result also has a probabilistic character: comparing with the Chung-Smirnov law of the iterated logarithm

$$
\limsup _{N \rightarrow \infty} \frac{N D_{N}\left(\xi_{1}, \ldots, \xi_{N}\right)}{\sqrt{2 N \log \log N}}=\frac{1}{2} \quad \text { a.s. }
$$

(see e.g. 17], p. 504), valid for uniformly distributed i.i.d. sequences $\left(\xi_{k}\right)_{k \geq 1}$, Philipp's result shows that the sequence $\left\langle n_{k} x\right\rangle$ behaves like a sequence of independent random variables. The probabilistic analogy is, however, not complete: the asymptotic properties of the sequence $\left\langle n_{k} x\right\rangle$ depend also on the number-theoretic properties of the sequence $\left(n_{k}\right)$ in an essential way. For example, Kac [12] showed that in the case $n_{k}=2^{k}$ the sequence $f\left(n_{k} x\right)$ satisfies the central limit theorem for all "nice" periodic functions $f$, and Gaposhkin [10] showed that this remains valid if the fractions $n_{k+1} / n_{k}$ are integers or if $n_{k+1} / n_{k} \rightarrow \alpha$, where $\alpha^{r}$ is irrational for $r=1,2, \ldots$. On the other hand, Erdős and Fortet (see [13, p. 646) observed that the central limit theorem for $f\left(n_{k} x\right)$ fails if $n_{k}=2^{k}-1$. As a consequence of the arithmetic connection, the limsup in (1.2) can be different from $1 / 2$, and it is still an open problem if the limsup is always a constant almost everywhere.

Very recently, Fukuyama 9] determined the limsup in (1.2) for the sequence $n_{k}=\theta^{k}, \theta>1$ (not necessarily integer). He showed that the limsup $\Sigma_{\theta}$ equals $1 / 2$ if $\theta^{r}$ is irrational for $r=1,2, \ldots$; i.e., in this case we get the same value as for an i.i.d. sequence. For other values of $\theta$, the dependence of the $\lim \sup$ on $\theta$ is very delicate. For example, Fukuyama showed that

$$
\begin{aligned}
& \Sigma_{\theta}=\sqrt{42} / 9, \quad \text { if } \theta=2, \\
& \Sigma_{\theta}=\frac{\sqrt{(\theta+1) \theta(\theta-2)}}{2 \sqrt{(\theta-1)^{3}}} \text { if } \theta \geq 4 \text { is an even integer, } \\
& \Sigma_{\theta}=\frac{\sqrt{\theta+1}}{2 \sqrt{\theta-1}} \text { if } \theta \geq 3 \text { is an odd integer. }
\end{aligned}
$$

It is worth observing that even though in the case $n_{k}=2^{k}$ the sequence $\left\langle n_{k} x\right\rangle$ obeys the central limit theorem by Kac's theorem, the lim sup in (1.2) differs from $1 / 2$, revealing a further delicacy of the problem. 
The purpose of our paper is to extend Fukuyama's results and to compute the limsup in (1.2) for a large class of integer sequences $\left(n_{k}\right)$. Noting that

$$
N D_{N}\left(n_{k} x\right)=\sup _{f \in \mathcal{A}}\left|\sum_{k=1}^{N} f\left(n_{k} x\right)\right|,
$$

where $\mathcal{A}$ denotes the class of functions $f=\mathbf{1}_{[a, b)}-(b-a), 0 \leq a<b \leq 1$, extended with period 1, a natural first step in the study of the LIL for $D_{N}\left(n_{k} x\right)$ is to investigate the asymptotic behavior of $\sum_{k=1}^{N} f\left(n_{k} x\right)$. By the classical theory, the limiting behavior of such sums is intimately connected with the number of solutions of the Diophantine equations

$$
a n_{k}-b n_{\ell}=\nu, \quad 1 \leq k, \ell \leq N
$$

In particular, Gaposhkin [1] showed that $f\left(n_{k} x\right)$ satisfies the CLT

$$
\lim _{N \rightarrow \infty} \mathbb{P}\left\{x \in(0,1): \sum_{k=1}^{N} f\left(n_{k} x\right) \leq t \sigma_{N}\right\}=(2 \pi)^{-1 / 2} \int_{-\infty}^{t} e^{-u^{2} / 2} d u
$$

with a suitable norming sequence $\sigma_{N}$ provided the number of solutions $(k, \ell)$ of (1.4) for nonzero integers $a, b, \nu$ is bounded by a constant $C(a, b)$ independent of $\nu$. Here, and in the sequel, $\mathbb{P}$ denotes the Lebesgue measure. This criterion covers the examples $n_{k+1} / n_{k} \rightarrow \infty$ and $n_{k+1} / n_{k} \rightarrow \alpha$, where $\alpha^{r} \notin \mathbb{Q}, r \geq 1$, but it is unnecessarily restrictive. Define, for $\left(n_{k}\right)_{k \geq 1}$ and $\nu \in \mathbb{Z}$,

$$
L(N, d, \nu)=\#\left\{1 \leq a, b \leq d, 1 \leq k, l \leq N: a n_{k}-b n_{l}=\nu\right\}
$$

and

$$
L(N, d)=\sup _{\nu>0} L(N, d, \nu), \quad L^{*}(N, d)=\sup _{\nu \geq 0} L(N, d, \nu) .
$$

For $\nu=0$ we do not count the trivial solutions $a=b, k=l$ in $L(N, d, \nu)$. In our recent paper [1] we showed that $f\left(n_{k} x\right)$ satisfies the CLT for all "nice" periodic functions $f$ provided for any $d \geq 1$,

$$
L(N, d)=o(N) \quad \text { as } N \rightarrow \infty,
$$

and this condition is optimal. Moreover, if we also assume that for any $d \geq 1$,

$$
L^{*}(N, d)=o(N) \quad \text { as } N \rightarrow \infty,
$$

then the CLT (1.5) holds with the norming sequence $\sigma_{N}=\|f\|_{2} \sqrt{N}$. In view of this precise characterization result, it is natural to expect that the value of the lim sup in (1.2) is also connected with the behavior of the Diophantine functions $L(N, d)$, $L^{*}(N, d)$. Our main result below shows that this is indeed the case and provides a near optimal criterion for the validity of the exact LIL for the discrepancy $D_{N}\left(n_{k} x\right)$ and the similar quantity $D_{N}^{*}\left(n_{k} x\right)$ ("star discrepancy"), where

$$
D_{N}^{*}=D_{N}^{*}\left(x_{1}, \ldots, x_{N}\right)=\sup _{0<a<1}\left|\frac{A(N, 0, a)}{N}-a\right| .
$$

Theorem 1.1. Let $\left(n_{k}\right)_{k \geq 1}$ be a sequence of positive integers satisfying the Hadamard gap condition (1.1), and assume that for any $d \geq 1$ we have

$$
L^{*}(N, d)=\mathcal{O}\left(\frac{N}{(\log N)^{1+\varepsilon}}\right) \quad \text { for some } \varepsilon>0 .
$$


Then

$$
\limsup _{N \rightarrow \infty} \frac{N D_{N}\left(n_{k} x\right)}{\sqrt{2 N \log \log N}}=\frac{1}{2} \quad \text { a.e. }
$$

and

$$
\limsup _{N \rightarrow \infty} \frac{N D_{N}^{*}\left(n_{k} x\right)}{\sqrt{2 N \log \log N}}=\frac{1}{2} \quad \text { a.e. }
$$

Clearly $L(N, d, \nu) \leq d^{2} N$ for all $N, d, \nu$, and therefore condition (1.8) is not far from optimal. We note that $\varepsilon$ in (1.8) is allowed to depend on $d$. It is possible that the conclusion of Theorem 1.1 remains valid if instead of (1.8) we assume only $L^{*}(N, d)=o(N)$, but on the basis of the difference between the conditions of the CLT and LIL for partial sums of independent random variables, it is more natural to expect that for the exact LIL for $D_{N}\left(n_{k} x\right)$ one needs a condition with at least a $\log \log N$ factor stronger than $L^{*}(N, d)=o(N)$. Note also that replacing $L^{*}(N, d)$ by $L(N, d)$ in (1.8), Theorem 1.1 becomes false: the modified condition is easily seen to hold for $n_{k}=2^{k}$, but by Fukuyama's result, the limsup in (1.2) is $\sqrt{42} / 9$ in this case.

It is easy to see that condition (1.8) and thus the exact LIL for $D_{N}\left(n_{k} x\right)$ is satisfied if $n_{k+1} / n_{k} \rightarrow \alpha$, where $\alpha^{r}$ is irrational for $r=1,2, \ldots$ or if $\left(n_{k}\right)$ satisfies the "large gap condition"

$$
n_{k+1} / n_{k} \rightarrow \infty \quad \text { as } \quad k \rightarrow \infty .
$$

As the following theorem shows, the exact LIL breaks down continuously if we weaken the growth condition (1.9). For $\left(n_{k}\right)$ satisfying the growth condition (1.1) for a "large" value of $q$, the limsup in the LIL for the discrepancy of $\left(n_{k} x\right)$ will be "almost" $1 / 2$. This falls in place with a result of Berkes [3], who found a similar phenomenon for sums $\sum f\left(n_{k} x\right)$ for "nice" periodic functions $f$. Writing

$$
\|f\|_{2}=\left(\int_{0}^{1} f(x)^{2} d x\right)^{1 / 2}
$$

he proved that for $\left(n_{k}\right)$ satisfying (1.1) for a "large" value of $q$ the lim sup of

$$
\left|\frac{\sum_{k=1}^{N} f\left(n_{k} x\right)}{2 N \log \log N}\right|
$$

will be "almost" $\|f\|_{2}$ a.e.

Theorem 1.2. Let $\left(n_{k}\right)_{k \geq 1}$ be a sequence of positive integers satisfying

$$
n_{k+1} / n_{k}>q \geq 2, \quad k=1,2, \ldots
$$

Then

$$
\left|\limsup _{N \rightarrow \infty} \frac{N D_{N}\left(n_{k} x\right)}{\sqrt{2 N \log \log N}}-\frac{1}{2}\right| \leq 8 q^{-1 / 4} \quad \text { a.e. }
$$

and

$$
\left|\limsup _{N \rightarrow \infty} \frac{N D_{N}^{*}\left(n_{k} x\right)}{\sqrt{2 N \log \log N}}-\frac{1}{2}\right| \leq 8 q^{-1 / 4} \quad \text { a.e. }
$$

Our next theorem, which is a by-product of the proof of Theorem 1.1 provides the law of the iterated logarithm for sums $\sum_{k=1}^{N} f\left(n_{k} x\right)$ under Diophantine conditions. We write $\operatorname{Var}_{[0,1]}(f)$ for the total variation of $f$ on the interval $[0,1]$. 
Theorem 1.3. Let $f$ be a function satisfying

$$
f(x+1)=f(x), \quad \int_{0}^{1} f(x) d x=0, \quad \operatorname{Var}_{[0,1]}(f)<\infty,
$$

and let $\left(n_{k}\right)$ be a sequence of integers satisfying the Hadamard gap condition (1.1) and the Diophantine condition (1.8) for any $d \geq 1$. Then

$$
\limsup _{N \rightarrow \infty} \frac{\left|\sum_{k=1}^{N} f\left(n_{k} x\right)\right|}{\sqrt{2 N \log \log N}}=\|f\|_{2} \quad \text { a.e. }
$$

Similarly to Theorem 1.1. the Diophantine condition in Theorem 1.3 is nearly optimal. Note that by Koksma's inequality (see e.g. [15], p. 143) the finiteness of the limsup in (1.11) follows from Philipp's LIL (1.2) and thus the essential new information provided by Theorem 1.3 is the exact value of the lim sup. It is worth pointing out that replacing $L^{*}$ by $L$ in (1.8) the value of the lim sup can be different from $\|f\|_{2}$, as the example $n_{k}=2^{k}$ shows; see Fukuyama [9].

\section{MAin Lemma}

The crucial step of the proof of Theorems 1.1 1.3 is Lemma 2.4 below, giving an exact LIL for lacunary trigonometric polynomials. With this lemma established, our theorems will be proved in Section 3 .

We start with some preliminary results. Here and in the sequel $\log x$ will stand for $\max \{1, \log x\}$.

Lemma 2.1 (Strassen 18). Let $\left(Y_{i}, \mathcal{F}_{i}, i \geq 1\right)$ be a martingale difference sequence with finite fourth moments, let $V_{M}=\sum_{i=1}^{M} \mathbb{E}\left(Y_{i}^{2} \mid \mathcal{F}_{i-1}\right)$ and assume $V_{1}=\mathbb{E} Y_{1}^{2}>0$ and $V_{M} \rightarrow \infty$. Assume additionally

$$
\lim _{M \rightarrow \infty} \frac{V_{M}}{s_{M}}=1 \quad \text { a.s. }
$$

with some positive sequence $s_{M}$, and

$$
\sum_{M=1}^{\infty} \frac{\left(\log s_{M}\right)^{10}}{s_{M}^{2}} \mathbb{E} Y_{M}^{4}<+\infty
$$

Then

$$
\limsup _{M \rightarrow \infty} \frac{\sum_{i=1}^{M} Y_{i}}{\sqrt{2 s_{M} \log \log s_{M}}}=1 \quad \text { a.s. }
$$

Condition (2.1) and the Beppo Levi theorem imply the a.s. convergence of the series

$$
\sum_{M=1}^{\infty} \frac{\left(\log s_{M}\right)^{10}}{s_{M}^{2}} \mathbb{E}\left(Y_{M}^{4} \mid \mathcal{F}_{M-1}\right)
$$

and hence by $V_{M} \sim s_{M}$ a.s. the series $\sum_{M=1}^{\infty} V_{M}^{-2}\left(\log V_{M}\right)^{10} \mathbb{E}\left(Y_{M}^{4} \mid \mathcal{F}_{M-1}\right)$ is also a.s. convergent. Thus

$$
\begin{aligned}
& \sum_{M=1}^{\infty} \frac{\left(\log V_{M}\right)^{5}}{V_{M}} \int_{x^{2}>V_{M}\left(\log V_{M}\right)^{-5}} x^{2} d P\left(Y_{M}<x \mid \mathcal{F}_{M-1}\right) \\
\leq & \sum_{M=1}^{\infty} \frac{\left(\log V_{M}\right)^{10}}{V_{M}^{2}} \int_{-\infty}^{+\infty} x^{4} d P\left(Y_{M}<x \mid \mathcal{F}_{M-1}\right)<+\infty \text { a.s. }
\end{aligned}
$$


Therefore by Corollary 4.5 of [18,

$$
\limsup _{M \rightarrow \infty} \frac{\sum_{i=1}^{M} Y_{i}}{\sqrt{2 V_{M} \log \log V_{M}}}=1 \quad \text { a.s., }
$$

and since $V_{M} \sim s_{M}$ a.s., this implies (2.2).

Lemma 2.2. For any function $f$ satisfying (1.10) we have

$$
\left|\int_{a}^{b} f(\lambda x) d x\right| \leq \frac{2}{\lambda} \int_{0}^{1}|f(x)| d x \leq \frac{2}{\lambda}\|f\|_{\infty}
$$

for any real numbers $a<b$ and any $\lambda>0$.

Proof. The lemma follows from

$$
\int_{a}^{b} f(\lambda x) d x=\frac{1}{\lambda} \int_{\lambda a}^{\lambda b} f(x) d x .
$$

Lemma 2.3 (Berkes, Philipp [5]). Let $\left(n_{k}\right)_{k \geq 1}$ be a sequence of positive integers satisfying the Hadamard gap condition and let $p(x)=\sum_{j=1}^{d}\left(a_{j} \cos 2 \pi j x+\right.$ $\left.b_{j} \sin 2 \pi j x\right)$ be a trigonometric polynomial of order $d$. Then

$$
\int_{0}^{1}\left(\sum_{k=N_{1}+1}^{N_{1}+N_{2}} p\left(n_{k} x\right)\right)^{4} d x \leq C N_{2}^{2}
$$

for all integers $N_{1}, N_{2} \geq 0$ and a number $C$ depending only on $p, d$ and $q$.

Lemma 2.4. Let $\left(n_{k}\right)_{k \geq 1}$ be a sequence of positive integers satisfying the Hadamard gap condition, let $d \geq 1$ and assume that (1.8) holds for some $\varepsilon>0$. Let $p(x)=$ $\sum_{j=1}^{d}\left(a_{j} \cos 2 \pi j x+b_{j} \sin 2 \pi j x\right)$ be a trigonometric polynomial of order $d$. Then

$$
\limsup _{N \rightarrow \infty} \frac{\left|\sum_{k=1}^{N} p\left(n_{k} x\right)\right|}{\sqrt{2 N \log \log N}}=\|p\|_{2} \quad \text { a.e. }
$$

Proof. We shall assume for the simplicity of writing that $p(x)$ is an even function; the proof in the general case is essentially the same. Thus let

$$
p(x)=\sum_{j=1}^{d} a_{j} \cos 2 \pi j x .
$$

We will assume that $\|p\|_{2}>0$, since otherwise the lemma is trivial. We will also assume without loss of generality that $\|p\|_{\infty} \leq 1$ and that $\left|a_{j}\right| \leq 1,1 \leq j \leq d$. Here, and in the rest of this section, $C$ will denote positive constants, not always the same, depending (at most) on $p, d$ and $q$.

We divide the set of positive integers into consecutive blocks

$$
\Delta_{1}^{\prime}, \Delta_{1}, \Delta_{2}^{\prime}, \Delta_{2}, \ldots, \Delta_{i}^{\prime}, \Delta_{i}, \ldots
$$

of lengths $\left\lceil 4 \log _{q} i\right\rceil$ and $i$, respectively. Letting $i^{-}$and $i^{+}$denote the smallest, resp. largest, integer in $\Delta_{i}$, we have

$$
\frac{n_{(i-1)^{+}}}{n_{i^{-}}} \leq q^{-4 \log _{q} i}=i^{-4}, \quad i \geq 2 .
$$


For every $k \in \bigcup_{i \geq 1} \Delta_{i}$, let $i=i(k)$ be defined by $k \in \Delta_{i}$, put $m(k)=\left\lceil\log _{2} n_{k}+\right.$ $\left.2 \log _{2} i\right\rceil$ and approximate $p\left(n_{k} x\right)$ by a discrete function $\varphi_{k}(x)$ such that the following properties are satisfied:

(P1) $\varphi_{k}(x)$ is constant for $\frac{v}{2^{m(k)}} \leq x<\frac{v+1}{2^{m(k)}}, \quad v=0,1, \ldots, 2^{m(k)}-1$;

(P2) $\left\|\varphi_{k}(x)-p\left(n_{k} x\right)\right\|_{\infty} \leq C i^{-2}$ Here

(P3) $\mathbb{E}\left(\varphi_{k}(x) \mid \mathcal{F}_{i-1}\right)=0$.

$$
Y_{i}=\sum_{k \in \Delta_{i}} \varphi_{k}(x)
$$

and $\mathcal{F}_{i}$ denotes the $\sigma$-field generated by the intervals

$$
\left[\frac{v}{2^{m\left(i^{+}\right)}}, \frac{v+1}{2^{m\left(i^{+}\right)}}\right), \quad 0 \leq v<2^{m\left(i^{+}\right)} .
$$

Since $p(x)$ is a trigonometric polynomial, it is Lipschitz continuous, and so

$$
\left|p\left(n_{k} x\right)-p\left(n_{k} x^{\prime}\right)\right| \leq C n_{k} 2^{-m(k)} \leq \frac{C}{i^{2}} \quad \text { for } \quad \frac{v}{2^{m(k)}} \leq x, x^{\prime} \leq \frac{v+1}{2^{m(k)}}, 0 \leq v<2^{m(k)} .
$$

Thus it is possible to approximate $p\left(n_{k} x\right)$ by discrete functions $\hat{\varphi}_{k}(x)$ that satisfy (P1) and (P2) only. Then for $k \in \Delta_{i}$ and any interval $I$ of the form $\left[\frac{v}{2^{m((i-1)+)}}\right.$, $\left.\frac{v+1}{2^{m\left((i-1)^{+}\right)}}\right)$for some $v$ we get, letting $|I|$ denote the lenght of $I$,

$$
\begin{aligned}
|I|^{-1}\left|\int_{I} \hat{\varphi}_{k}(x) d x\right| & \leq|I|^{-1}\left(\left|\int_{I} p\left(n_{k} x\right) d x\right|+\int_{I} C i^{-2} d x\right) \\
& \leq \frac{2\|p\|_{\infty} 2^{m\left((i-1)^{+}\right)}}{n_{i^{-}}}+C i^{-2} \\
& \leq \frac{4 i^{2} n_{(i-1)^{+}}}{n_{i^{-}}}+C i^{-2} \\
& \leq C i^{-2}
\end{aligned}
$$

by (2.3), Lemma 2.2 and since $\|p\|_{\infty} \leq 1$. For every $x \in[0,1)$ we can find an interval of type $I$ for some $v$ such that $x \in I$, and we put $\varphi_{k}(x)=\hat{\varphi}_{k}(x)-|I|^{-1} \int_{I} \hat{\varphi}_{k}(t) d t$. Then these functions $\varphi_{k}(x)$ satisfy (P1), (P2) and (P3).

We put

$$
T_{i}=\sum_{k \in \Delta_{i}} p\left(n_{k} x\right) \quad T_{i}^{\prime}=\sum_{k \in \Delta_{i}^{\prime}} p\left(n_{k} x\right), \quad V_{M}=\sum_{i=1}^{M} \mathbb{E}\left(Y_{i}^{2} \mid \mathcal{F}_{i-1}\right) .
$$

We put

$$
s_{M}=\|p\|_{2}^{2} \sum_{i=1}^{M}\left|\Delta_{i}\right|
$$


where $\left|\Delta_{i}\right|$ denotes the number of integers in $\Delta_{i}$. For given $M$ we want to estimate $\left\|V_{M}-s_{M}\right\|_{2}$. We have

$$
\begin{aligned}
& T_{i}(x)^{2}-\|p\|_{2}^{2}\left|\Delta_{i}\right| \\
& =\left(\sum_{k \in \Delta_{i}} p\left(n_{k} x\right) d x\right)^{2}-\|p\|_{2}^{2}\left|\Delta_{i}\right| \\
& =\left(\sum_{k \in \Delta_{i}} \sum_{j=1}^{d} a_{j} \cos 2 \pi j n_{k} x\right)^{2}-\left(\frac{1}{2} \sum_{i=1}^{d} a_{j}^{2}\right)\left|\Delta_{i}\right| \\
& =\sum_{\substack{1 \leq j, j^{\prime} \leq d, k, k^{\prime} \in \Delta_{i},(j, k) \neq\left(j^{\prime}, k^{\prime}\right) \\
0 \leq\left|j n_{k}-j^{\prime} n_{k^{\prime}}\right| \leq n_{(i-1)^{+}}}} \frac{1}{2} a_{j} a_{j^{\prime}} \cos 2 \pi\left(j n_{k}-j^{\prime} n_{k^{\prime}}\right) x \\
& +\sum_{1 \leq j, j^{\prime} \leq d, k, k^{\prime} \in \Delta_{i}} \frac{1}{2} a_{j} a_{j^{\prime}} \cos 2 \pi\left(j n_{k}-j^{\prime} n_{k^{\prime}}\right) x \\
& n_{(i-1)}+<\left|j n_{k}-j^{\prime} n_{k^{\prime}}\right|<n_{i-} \\
& +R_{i}(x) \\
& (2.4)=U_{i}(x)+W_{i}(x)+R_{i}(x) \text {. }
\end{aligned}
$$

Since $R_{i}(x)$ is a sum of at most $2 d^{2}\left|\Delta_{i}\right|^{2}$ trigonometric functions with coefficients at most 1 and frequencies at least $n_{i^{-}}$, by Lemma 2.2 .

$$
\left|\mathbb{E}\left(R_{i} \mid \mathcal{F}_{i-1}\right)\right| \leq 4 d^{2}\left|\Delta_{i}\right|^{2} \frac{2^{m\left((i-1)^{+}\right)}}{n_{i^{-}}} \leq C
$$

and consequently

$$
\left|\sum_{i=1}^{M} \mathbb{E}\left(R_{i} \mid \mathcal{F}_{i-1}\right)\right| \leq C M .
$$

$U_{i}$ and $W_{i}$ are sums of at most $C i$ trigonometric functions with coefficients at most 1. Indeed, the number of quadruples $\left(j, j^{\prime}, k, k^{\prime}\right)$ with $1 \leq j, j^{\prime} \leq d, k, k^{\prime} \in \Delta_{i}$, for which $\left|j n_{k}-j^{\prime} n_{k^{\prime}}\right|<n_{i^{-}}$is at most $C\left|\Delta_{i}\right|$, since for fixed $j, j^{\prime}$ and $k$ in the case $n_{k^{\prime}}>(d+1) n_{k}$ we have $j n_{k}-j^{\prime} n_{k^{\prime}} \leq j n_{k}-j^{\prime}(d+1) n_{k} \leq(d-(d+1)) n_{k}=-n_{k} \leq$ $-n_{i^{-}}$and there are at most $1+\log _{q}(d+1)$ indices $k^{\prime}>k$ for which $n_{k^{\prime}} \leq(d+1) n_{k}$. In particular,

$$
\left\|U_{i}\right\|_{\infty} \leq C i \quad \text { and } \quad\left\|W_{i}\right\|_{\infty} \leq C i
$$

By (2.4), (2.5), Minkowski's inequality and

$$
\left\|Y_{i}^{2}-T_{i}^{2}\right\|_{\infty} \leq\left\|Y_{i}-T_{i}\right\|_{\infty}\left\|Y_{i}+T_{i}\right\|_{\infty} \leq C\left|\Delta_{i}\right| i^{-2}\left|\Delta_{i}\right| \leq C,
$$

we get

$$
\begin{aligned}
& \left\|V_{M}-s_{M}\right\|_{2} \\
\leq & \left\|\sum_{i=1}^{M}\left(\mathbb{E}\left(T_{i}^{2} \mid \mathcal{F}_{i-1}\right)-\|p\|_{2}^{2}\left|\Delta_{i}\right|\right)\right\|_{2}+C M \\
\leq & \left\|\sum_{i=1}^{M} \mathbb{E}\left(U_{i} \mid \mathcal{F}_{i-1}\right)\right\|_{2}+\left\|\sum_{i=1}^{M} \mathbb{E}\left(W_{i} \mid \mathcal{F}_{i-1}\right)\right\|_{2}+C M .
\end{aligned}
$$


To estimate $\left\|\sum_{i=1}^{M} \mathbb{E}\left(W_{i} \mid \mathcal{F}_{i-1}\right)\right\|_{2}$, we observe

$$
\left(\sum_{i=1}^{M} \mathbb{E}\left(W_{i} \mid \mathcal{F}_{i-1}\right)\right)^{2} \leq 2 \sum_{1 \leq i \leq i \leq M} \mathbb{E}\left(W_{i} \mid \mathcal{F}_{i-1}\right) \mathbb{E}\left(W_{i^{\prime}} \mid \mathcal{F}_{i^{\prime}-1}\right)
$$

By (2.6),

$$
\sum_{i=1}^{M}\left(\mathbb{E}\left(W_{i} \mid \mathcal{F}_{i-1}\right)\right)^{2} \leq \sum_{i=1}^{M} C i^{2} \leq C M^{3} .
$$

For $i<i^{\prime}$, since $\mathbb{E}\left(W_{i} \mid \mathcal{F}_{i-1}\right)$ is $\mathcal{F}_{i-1}$-measurable,

$$
\begin{aligned}
\left|\mathbb{E}\left(\mathbb{E}\left(W_{i} \mid \mathcal{F}_{i-1}\right) \mathbb{E}\left(W_{i^{\prime}} \mid \mathcal{F}_{i^{\prime}-1}\right) \mid \mathcal{F}_{i-1}\right)\right| & =\left|\mathbb{E}\left(W_{i} \mid \mathcal{F}_{i-1}\right) \mathbb{E}\left(W_{i^{\prime}} \mid \mathcal{F}_{i-1}\right)\right| \\
& \leq\left\|W_{i}\right\|_{\infty}\left|\mathbb{E}\left(W_{i^{\prime}} \mid \mathcal{F}_{i-1}\right)\right| \\
& \leq C i\left|\mathbb{E}\left(W_{i^{\prime}} \mid \mathcal{F}_{i-1}\right)\right|
\end{aligned}
$$

and thus

$$
\left|\mathbb{E}\left(\mathbb{E}\left(W_{i} \mid \mathcal{F}_{i-1}\right) \mathbb{E}\left(W_{i^{\prime}} \mid \mathcal{F}_{i^{\prime}-1}\right)\right)\right| \leq C i \mathbb{E}\left|\mathbb{E}\left(W_{i^{\prime}} \mid \mathcal{F}_{i-1}\right)\right| .
$$

$W_{i^{\prime}}$ can be written as a trigonometric polynomial of the form

$$
\sum_{u=n_{\left(i^{\prime}-1\right)^{+}}}^{n_{i^{\prime}-}} c_{u} \cos 2 \pi u x
$$

where $\sum_{u}\left|c_{u}\right| \leq C\left|\Delta_{i^{\prime}}\right|$. Thus using Lemma 2.2 with $f(x)=\cos 2 \pi x$ we get

$$
\begin{aligned}
\left|\mathbb{E}\left(W_{i^{\prime}} \mid \mathcal{F}_{i-1}\right)\right| & \leq \sum_{u=n_{\left(i^{\prime}-1\right)^{+}}}^{n_{i^{\prime}-}}\left|c_{u}\right| u^{-1} 2^{m\left((i-1)^{+}\right)} \\
& \leq C i^{\prime} \frac{i^{2} n_{(i-1)^{+}}}{n_{\left(i^{\prime}-1\right)^{+}}} \\
& \leq C i^{2} i^{\prime} q^{(i-1)^{+}-\left(i^{\prime}-1\right)^{+}} \leq C i^{2} i^{\prime} q^{-\left(i^{\prime}-1\right)} .
\end{aligned}
$$

Combining the estimates (2.9), (2.10) and (2.11) we see that

$$
\left\|\sum_{i=1}^{M} \mathbb{E}\left(W_{i} \mid \mathcal{F}_{i-1}\right)\right\|_{2}=\left(C M^{3}+\sum_{1 \leq i<i^{\prime} \leq M} C i^{3} i^{\prime} q^{-\left(i^{\prime}-1\right)}\right)^{1 / 2} \leq C M^{3 / 2} .
$$

To estimate $\left\|\sum_{i=1}^{M} \mathbb{E}\left(U_{i} \mid \mathcal{F}_{i-1}\right)\right\|_{2}$, we note that $U_{i}$ is a sum of trigonometric functions with frequencies at most $n_{(i-1)^{+}}$; i.e.,

$$
U_{i}=\sum_{u=0}^{n_{(i-1)+}^{+}} c_{u} \cos 2 \pi u x
$$

where $\sum_{u}\left|c_{u}\right| \leq C\left|\Delta_{i}\right|$. Hence the fluctuation of $U_{i}$ on any atom of $\mathcal{F}_{i-1}$ is at most

$$
\sum_{u=0}^{n_{(i-1)^{+}}}\left|c_{u}\right| 2 \pi u 2^{-m\left((i-1)^{+}\right)} \leq C i \frac{n_{(i-1)^{+}}}{i^{2} n_{(i-1)^{+}}} \leq C i^{-1},
$$

and consequently

$$
\left|\mathbb{E}\left(U_{i} \mid \mathcal{F}_{i-1}\right)-U_{i}\right| \leq C i^{-1}
$$


which gives

$$
\left\|\sum_{i=1}^{M} E\left(U_{i} \mid \mathcal{F}_{i-1}\right)\right\|_{2} \leq\left\|\sum_{i=1}^{M} U_{i}\right\|_{2}+C \log M .
$$

The largest frequency of a trigonometric function in $\sum_{i=1}^{M} U_{i}$ is at most $n_{(M-1)^{+}}$, so we can write

$$
\sum_{i=1}^{M} U_{i}(x)=\sum_{u=0}^{n_{(M-1)+}} c_{u} \cos 2 \pi u x
$$

where by the Diophantine condition (1.8),

$$
\left|c_{u}\right| \leq C \frac{\sum_{i=1}^{M}\left(\left|\Delta_{i}\right|+\left|\Delta_{i^{\prime}}\right|\right)}{\left(\log \left(\sum_{i=1}^{M}\left|\Delta_{i}\right|+\left|\Delta_{i^{\prime}}\right|\right)\right)^{1+\varepsilon}} \leq C M^{2}(\log M)^{-(1+\varepsilon)},
$$

and $\sum_{u}\left|c_{u}\right| \leq C \sum_{i=1}^{M}\left|\Delta_{i}\right| \leq C M^{2}$. Thus, using (2.13), we get

$$
\begin{aligned}
\left\|\sum_{i=1}^{M} E\left(U_{i} \mid \mathcal{F}_{i-1}\right)\right\|_{2} & \leq\left(\sum_{u=0}^{n_{(M-1)+}} c_{u}^{2}\right)^{1 / 2}+C \log M \\
& \leq\left(C M^{2} M^{2}(\log M)^{-(1+\varepsilon)}\right)^{1 / 2}+C \log M \\
& \leq C M^{2}(\log M)^{-(1+\varepsilon) / 2} .
\end{aligned}
$$

Substituting the estimates (2.12) and (2.14) into (2.8), we obtain

$$
\left\|V_{M}-s_{M}\right\|_{2} \leq C M^{2}(\log M)^{-(1+\varepsilon) / 2} .
$$

We choose an $\alpha>0$ such that

$$
\left(1+\frac{\varepsilon}{2}\right)^{-1}<\alpha<\left(1+\frac{\varepsilon}{4}\right)^{-1}
$$

and define numbers

$$
M_{l}=\left\lfloor 2^{\left(l^{\alpha}\right)}\right\rfloor, \quad l \geq 0
$$

and sets

$$
S_{l}=\bigcup_{M_{l} \leq M \leq M_{l+1}}\left\{x \in(0,1):\left|V_{M}-s_{M}\right|>\frac{s_{M}}{(\log M)^{\varepsilon / 4}}\right\}, \quad l \geq 0 .
$$

Then, since $\alpha<1$,

$$
\lim _{l \rightarrow \infty} s_{M_{l+1}} / s_{M_{l}}=1
$$

and

$$
l^{\alpha-1}\left(\log M_{l}\right)^{\varepsilon / 4} \leq C l^{\alpha-1}\left(l^{\alpha}\right)^{\varepsilon / 4} \rightarrow 0 \quad \text { as } \quad l \rightarrow \infty .
$$

Since $V_{M}$ and $s_{M}$ are increasing in $M$, and since for $l \geq 1$,

$$
\begin{aligned}
s_{M_{l+1}}-s_{M_{l}} & =s_{M_{l}}\left(\frac{s_{M_{l+1}}}{s_{M_{l}}}-1\right) \\
& \leq s_{M_{l}}\left(C \frac{2^{2(l+1)^{\alpha}}}{2^{2\left(l^{\alpha}\right)}}-1\right) \leq s_{M_{l}}\left(C 2^{2 \alpha l^{\alpha-1}}-1\right) \leq C s_{M_{l}} l^{\alpha-1}
\end{aligned}
$$


we get, using (2.17) and (2.18), that

$$
\begin{aligned}
\mathbb{P}\left(S_{l}\right) \leq & \mathbb{P}\left\{V_{M_{l}}-s_{M_{l+1}}<-\frac{s_{M_{l}}}{\left(\log M_{l+1}\right)^{\varepsilon / 4}}\right\}+\mathbb{P}\left\{V_{M_{l+1}}-s_{M_{l}}>\frac{s_{M_{l}}}{\left(\log M_{l+1}\right)^{\varepsilon / 4}}\right\} \\
\leq & \mathbb{P}\left\{V_{M_{l}}-s_{M_{l}}<C s_{M_{l}} l^{\alpha-1}-C \frac{s_{M_{l}}}{\left(\log M_{l}\right)^{\varepsilon / 4}}\right\} \\
& +\mathbb{P}\left\{V_{M_{l+1}}-s_{M_{l+1}}>-C s_{M_{l}} l^{\alpha-1}+C \frac{s_{M_{l+1}}}{\left(\log M_{l+1}\right)^{\varepsilon / 4}}\right\} \\
\leq & \mathbb{P}\left\{\left|V_{M_{l}}-s_{M_{l}}\right|>C \frac{s_{M_{l}}}{\left(\log M_{l}\right)^{\varepsilon / 4}}\right\} \\
& +\mathbb{P}\left\{\left|V_{M_{l+1}}-s_{M_{l+1}}\right|>C \frac{s_{M_{l+1}}}{\left(\log M_{l+1}\right)^{\varepsilon / 4}}\right\} \\
\leq & C \frac{1}{\left(l^{\alpha}\right)^{1+\varepsilon / 2}},
\end{aligned}
$$

for sufficiently large $l$, where the last inequality follows from (2.15) and Chebyshev's inequality. Therefore by (2.16),

$$
\sum_{l=1}^{\infty} \mathbb{P}\left(S_{l}\right)<+\infty
$$

and thus the Borel-Cantelli Lemma implies that the set of those $x \in(0,1)$, that are contained in infinitely many sets $S_{l}, l \geq 1$, has Lebesgue measure 0 . This implies $V_{M} \sim s_{M}$ a.e.

By Lemma 2.3 and (2.7),

$$
\mathbb{E} Y_{M}^{4} \leq C\left|\Delta_{M}\right|^{2} \leq C M^{2}
$$

and thus by $C M^{2} \leq s_{M} \leq C M^{2}$ we have

$$
\sum_{M=1}^{\infty} \frac{\left(\log s_{M}\right)^{10}}{s_{M}^{2}} \mathbb{E} Y_{M}^{4} \leq \sum_{M=1}^{\infty} C \frac{(\log M)^{10}}{M^{2}}<+\infty .
$$

Hence by Lemma 2.1.

$$
\limsup _{M \rightarrow \infty} \frac{\left|\sum_{i=1}^{M} Y_{i}\right|}{\sqrt{2 s_{M} \log \log s_{M}}}=1 \quad \text { a.e. }
$$

We add the sum of the "short blocks" $T_{i}^{\prime}$, for which

$$
\left|\sum_{i=1}^{M} T_{i}^{\prime}\right|=\mathcal{O}(\sqrt{M(\log M) \log \log (M \log M)}) \quad \text { a.e. }
$$

by (1.2) and Koksma's inequality, change from $Y_{i}$ to $T_{i}$, where $\left|Y_{i}-T_{i}\right| \leq C\left|\Delta_{i}\right| i^{-2} \leq$ $C i^{-1}$ by part of (2.7), and get

$$
\limsup _{M \rightarrow \infty} \frac{\left|\sum_{k=1}^{M^{+}} p\left(n_{k} x\right)\right|}{\sqrt{2 s_{M} \log \log s_{M}}}=\limsup _{M \rightarrow \infty} \frac{\left|\sum_{i=1}^{M}\left(T_{i}+T_{i}^{\prime}\right)\right|}{\sqrt{2 s_{M} \log \log s_{M}}}=1 \quad \text { a.e. }
$$

Now we want to break into the blocks of sums. Since

$$
\max _{N \in \Delta_{i} \cup \Delta_{i}^{\prime}}\left|\sum_{k \in \Delta_{i} \cup \Delta_{i}^{\prime}, k \leq N} p\left(n_{k} x\right)\right| \leq C\left(\left|\Delta_{i}\right|+\left|\Delta_{i}^{\prime}\right|\right) \leq C i,
$$


it follows that

$$
\limsup _{M \rightarrow \infty} \frac{\left|\max _{(M-1)^{+}<N \leq M^{+}} \sum_{k \leq N} p\left(n_{k} x\right)\right|}{\sqrt{2 s_{M} \log \log s_{M}}}=1 \quad \text { a.e. }
$$

For $N \geq 1$ we define $M(N)$ as the index $m$, for which $N$ is contained in $\Delta_{m} \cup \Delta_{m}^{\prime}$. Then

$$
\limsup _{N \rightarrow \infty} \frac{\left|\sum_{k \leq N} p\left(n_{k} x\right)\right|}{\sqrt{2 s_{M(N)} \log \log s_{M(N)}}}=1 \quad \text { a.e. }
$$

Since

$$
\frac{s_{M(N)}}{N} \rightarrow\|p\|_{2}^{2} \quad \text { as } \quad N \rightarrow \infty
$$

Lemma 2.4 follows.

\section{Proof of Theorems $1.1-1.3$}

To prove Theorem 1.3 we first show

Lemma 3.1. Let $\left(n_{k}\right)_{k \geq 1}$ be a sequence of positive integers satisfying the Hadamard gap condition, and let $r(x)$ be a function of the form

$$
r(x)=\sum_{j=d+1}^{\infty}\left(a_{j} \cos 2 \pi j x+b_{j} \sin 2 \pi j x\right),
$$

for some fixed $d \geq 0$, where

$$
\left|a_{j}\right| \leq j^{-1} \quad \text { and } \quad\left|b_{j}\right| \leq j^{-1}, \quad j \geq d+1 .
$$

Then

$$
\limsup _{N \rightarrow \infty} \frac{\left|\sum_{k=1}^{N} r\left(n_{k} x\right)\right|}{\sqrt{2 N \log \log N}} \leq C_{q} d^{-1 / 4} \quad \text { a.e., }
$$

where $C_{q}$ is a positive number depending on $q$. For $q \geq 2$ we can choose $C_{q}=5$.

Note that Lemma 3.1 is valid without any Diophantine conditions.

Proof. The proof of Lemma 3.1 can be easily modelled after [19] and [16]. Combining the argument in [19, p. 104] and Lemma 3 of [16, p. 246] proves

$$
\limsup _{N \rightarrow \infty} \frac{\left|\sum_{k=1}^{N} r\left(n_{k} x\right)\right|}{\sqrt{N \log \log N}} \leq 3 \sqrt{2 B} \quad \text { a.e. }
$$

where

$$
B=\left(q^{1 / 2}-1\right)^{-1}\|r\|_{2}
$$

Takahashi's paper considers only Lipschitz continuous functions, but the proof remains valid without any change for functions satisfying the conditions of Lemma 3.1. Clearly

$$
\|r\|_{2}^{2}=\frac{1}{2} \sum_{j=d+1}^{\infty}\left(a_{j}^{2}+b_{j}^{2}\right) \leq \sum_{j=d+1}^{\infty} j^{-2} \leq d^{-1},
$$

completing the proof. 
Proof of Theorem 1.3. Without loss of generality we can assume $\operatorname{Var}_{[0,1]}(f) \leq 2$. Letting

$$
f(x) \sim \sum_{j=1}^{\infty}\left(a_{j} \cos 2 \pi j x+b_{j} \sin 2 \pi j x\right)
$$

denote the Fourier series of $f$, we have (see Zygmund [21, p. 48])

$$
\left|a_{j}\right| \leq 1 / j, \quad\left|b_{j}\right| \leq 1 / j, \quad j \geq 1 .
$$

Thus for any fixed $d \geq 1, f(x)$ can be written as a sum of a trigonometric polynomial $p(x)$ of order $d$ and a remainder function $r(x)$ satisfying the conditions of Lemma 3.1. Applying Lemma 2.4 and Lemma 3.1 we get

$$
\limsup _{N \rightarrow \infty} \frac{\left|\sum_{k=1}^{N} f\left(n_{k} x\right)\right|}{\sqrt{2 N \log \log N}} \leq\|p\|_{2}+C_{q} d^{-1 / 2} \leq\|f\|_{2}+C_{q} d^{-1 / 2} \quad \text { a.e. }
$$

and

$$
\limsup _{N \rightarrow \infty} \frac{\left|\sum_{k=1}^{N} f\left(n_{k} x\right)\right|}{\sqrt{2 N \log \log N}} \geq\|p\|_{2}-C_{q} d^{-1 / 2} \geq\|f\|_{2}-\left(C_{q}+1\right) d^{-1 / 2} \quad \text { a.e., }
$$

since

$$
\|p\|_{2} \geq\|f\|_{2}-\|r\|_{2} \geq\|f\|_{2}-d^{-1 / 2} .
$$

Since $d$ can be chosen arbitrarily, Theorem 1.3 follows.

To prove Theorems 1.1 and 1.2 we define, for $r \geq 0, N \geq 1$ and $\left(x_{1}, \ldots, x_{N}\right) \in$ $\mathbb{R}^{N}$

$$
\begin{gathered}
D_{N}^{\left(\leq 2^{-r}\right)}\left(x_{1}, \ldots, x_{N}\right)=\sup _{0 \leq a<b \leq 1, b-a \leq 2^{-r}}\left|\frac{\sum_{k=1}^{N} \mathbf{I}_{[a, b)}\left(x_{k}\right)}{N}\right|, \\
D_{N}^{\left(\geq 2^{-r}\right)}\left(x_{1}, \ldots, x_{N}\right)=\max _{a_{1}, a_{2} \in \mathbb{Z}, 0 \leq a_{1}<a_{2} \leq 2^{r}}\left|\frac{\sum_{k=1}^{N} \mathbf{I}_{\left[a_{1} 2^{-r}, a_{2} 2^{-r}\right)}\left(x_{k}\right)}{N}\right|,
\end{gathered}
$$

and

$$
D_{N}^{*}{ }^{\left(\geq 2^{-r}\right)}\left(x_{1}, \ldots, x_{N}\right)=\max _{a_{1} \in \mathbb{Z}, 0<a_{1}<2^{r}}\left|\frac{\sum_{k=1}^{N} \mathbf{I}_{\left[0, a_{1} 2^{-r}\right)}\left(x_{k}\right)}{N}\right|,
$$

where $\mathbf{I}_{[a, b)}$ denotes the indicator of the interval $[a, b)$, extended with period 1 and centered at expectation, i.e.

$$
\mathbf{I}_{[a, b)}(x)=\mathbf{1}_{[a, b)}(\langle x\rangle)-(b-a) .
$$

It is easy to see that

$$
D_{N}^{\left(\geq^{-r}\right)} \leq D_{N} \leq D_{N}^{\left(\geq^{-r}\right)}+2 D_{N}^{\left(\leq 2^{-r}\right)}
$$

and

$$
D_{N}^{*}\left(2^{-r}\right) \leq D_{N}^{*} \leq D_{N}
$$

The idea to split the discrepancy $D_{N}$ into two parts $D_{N}^{\left(\leq 2^{-r}\right)}$ and $D_{N}^{\left(\geq 2^{-r}\right)}$ to prove an exact LIL for the discrepancy of $\left(n_{k} x\right)$ is due to Fukuyama [9]. 
Lemma 3.2. Let $\left(n_{k}\right)_{k \geq 1}$ be a sequence of positive integers satisfying the Hadamard gap condition and the Diophantine condition (1.8) for any $d \geq 1$. Then for any fixed $r \geq 0$,

and

$$
\limsup _{N \rightarrow \infty} \frac{N D_{N}^{\left(\geq 2^{-r}\right)}\left(n_{k} x\right)}{\sqrt{2 N \log \log N}}=\frac{1}{2} \quad \text { a.e. }
$$

$$
\limsup _{N \rightarrow \infty} \frac{N D_{N}^{*}\left(2^{-r}\right)\left(n_{k} x\right)}{\sqrt{2 N \log \log N}}=\frac{1}{2} \quad \text { a.e. }
$$

Proof. Clearly, $\mathbf{I}_{[a, b)}(x), 0 \leq a<b \leq 1$ satisfies (1.10). Thus Lemma 3.2 follows from Theorem 1.3. the definitions of $D_{N}^{\left(\geq^{-r}\right)}$ and $D_{N}^{\left(\leq 2^{-r}\right)}$ and the fact that

$$
\max _{a_{1}, a_{2} \in \mathbb{Z}, 0 \leq a_{1}<a_{2} \leq 2^{r}}\left\|\mathbf{I}_{\left[a_{1} 2^{-r}, a_{2} 2^{-r}\right)}\right\|_{2}=\left\|\mathbf{I}_{[0,1 / 2)}\right\|_{2}=1 / 2
$$

and

$$
\max _{a_{1} \in \mathbb{Z}, 0<a_{1}<2^{r}}\left\|\mathbf{I}_{\left[0, a_{1} 2^{-r}\right)}\right\|_{2}=\left\|\mathbf{I}_{[0,1 / 2)}\right\|_{2}=1 / 2 .
$$

Lemma 3.3. Let $\left(n_{k}\right)_{k \geq 1}$ be a sequence of positive integers satisfying the Hadamard gap condition. Then for any $r \geq 1$,

$$
\limsup _{N \rightarrow \infty} \frac{N D_{N}^{\left(\leq 2^{-r}\right)}\left(n_{k} x\right)}{\sqrt{2 N \log \log N}} \leq C_{q} r^{-1} \quad \text { a.e., }
$$

where $C_{q}$ is a positive number depending on $q$. In particular,

$$
\lim _{r \rightarrow \infty} \limsup _{N \rightarrow \infty} \frac{N D_{N}^{\left(\leq 2^{-r}\right)}\left(n_{k} x\right)}{\sqrt{2 N \log \log N}}=0 \quad \text { a.e. }
$$

Similarly to Lemma 3.1, this lemma is valid without any Diophantine conditions.

Proof. The proof of this lemma can be modelled after [16]. It remains completely the same up to the end of page 249. On page 250, Philipp shows that

$$
\limsup _{N \rightarrow \infty} \sup _{a \in[0,1)} \frac{\sum_{k=1}^{N} \mathbf{I}_{[0, a)}\left(n_{k} x\right)}{\sqrt{N \log \log N}} \leq C_{q} \quad \text { a.e., }
$$

where $C_{q}$ is a positive number depending on $q$. Under the additional assumption that $a \leq 2^{-r}$ for fixed $r \geq 1$, it is easy to see that in the first equation on page 250 it suffices to sum over $h$ from $r$ to $H$ instead of 1 to $H$. Thus we get

$$
\limsup _{N \rightarrow \infty} \sup _{a \in\left[0,2^{-r}\right)} \frac{\sum_{k=1}^{N} \mathbf{I}_{[0, a)}\left(n_{k} x\right)}{\sqrt{N \log \log N}} \leq C_{q} \sum_{h=r}^{\infty} 2^{-h / 8} \text { a.e. }
$$

Using the same method it is easy to see that a similar result holds for "shifted intervals", i.e.

$$
\limsup _{N \rightarrow \infty} \sup _{a \in\left[0,2^{-r}\right)} \frac{\sum_{k=1}^{N} \mathbf{I}_{\left[l 2^{-r}, 2^{-r}+a\right)}\left(n_{k} x\right)}{\sqrt{N \log \log N}} \leq C_{q} \sum_{h=r}^{\infty} 2^{-h / 8} \text { a.e., }
$$

for any integer $l$ satisfying $0 \leq l \leq 2^{r}-1$, and, using the same argument in a mirror-inverted way, that

$$
\limsup _{N \rightarrow \infty} \sup _{a \in\left[0,2^{-r}\right)} \frac{\sum_{k=1}^{N} \mathbf{I}_{\left[l 2^{-r}-a, l 2^{-r}\right)}\left(n_{k} x\right)}{\sqrt{N \log \log N}} \leq C_{q} \sum_{h=r}^{\infty} 2^{-h / 8} \text { a.e., }
$$


where $1 \leq l \leq 2^{r}$. Those $x \in[0,1$ ), for which (3.3) and (3.4) are valid for all possible values of $l$, satisfy

$$
\limsup _{N \rightarrow \infty} \sup _{0 \leq a<b<1, b-a \leq 2^{-r}} \frac{\sum_{k=1}^{N} \mathbf{I}_{[a, b)}\left(n_{k} x\right)}{\sqrt{N \log \log N}} \leq 2 C_{q} \sum_{h=r}^{\infty} 2^{-h / 8} \quad \text { a.e. }
$$

as well. Since there are only finitely many possibilities to choose $l$ in (3.3) and (3.4), the Lebesgue measure of the exceptional set in (3.5) has to be zero as well. This, together with the fact that

$$
\sum_{h=r}^{\infty} 2^{-h / 8} \leq C r^{-1}
$$

with an absolute constant $C$, proves Lemma 3.3

Proof of Theorem 1.1. Theorem 1.1 follows from Lemma 3.2 and Lemma 3.3, together with (3.1) and (3.2).

Proof of Theorem 1.2. Theorem 1.2 follows from Lemma 2.4. Lemma 3.1 and Lemma 3.3. Assume $\left(n_{k}\right)$ is a sequence of positive integers such that

$$
n_{k+1} / n_{k}>q \geq 2, \quad k \geq 1 .
$$

Set $d=\lfloor q / 2\rfloor$. Then for fixed $1 \leq j, j^{\prime} \leq d$, the sequence

$$
\left(m_{k}\right)_{k \geq 1}=\left(m_{k}\left(j, j^{\prime}\right)_{k \geq 1}=\left(j n_{k}\right)_{k \geq 1} \cup\left(j^{\prime} n_{k}\right)_{k \geq 1},\right.
$$

i.e. the sequence consisting of the elements of the set-theoretic union of $\left(j n_{k}\right)_{k \geq 1}$ and $\left(j^{\prime} n_{k}\right)_{k \geq 1}$, sorted in increasing order, is lacunary. Now by a well-known property of lacunary sequences (see Zygmund [21, p. 203]), this implies that the number of solutions of the Diophantine equation

$$
m_{k}-m_{k^{\prime}}=\nu, \quad k, k^{\prime} \geq 1
$$

is bounded by a number $C\left(j, j^{\prime}\right)$, uniformly in $\nu \in \mathbb{Z}, \nu \neq 0$. Also, the number of solutions of

$$
j n_{k}-j^{\prime} n_{k^{\prime}}=0, \quad k, k^{\prime} \geq 1
$$

is bounded by $C\left(j, j^{\prime}\right)$. Thus the sequence $\left(n_{k}\right)_{k \geq 1}$ satisfies (1.8) for this choice of $d$. By Lemma 2.4 and Lemma 3.1, for any function $f(x)$ satisfying (1.10), writing $p(x)$ for the $d$-th partial sum of $f(x)$,

$$
\limsup _{N \rightarrow \infty} \frac{\left|\sum_{k=1}^{N} f\left(n_{k} x\right)\right|}{\sqrt{2 N \log \log N}} \leq\|p\|_{2}+5 d^{-1 / 4} \leq\|f\|_{2}+6 q^{-1 / 4} \quad \text { a.e. }
$$

and similarly

$$
\limsup _{N \rightarrow \infty} \frac{\left|\sum_{k=1}^{N} f\left(n_{k} x\right)\right|}{\sqrt{2 N \log \log N}} \geq\|p\|_{2}-5 d^{-1 / 4} \geq\|f\|_{2}-6 d^{-1 / 4} \geq\|f\|_{2}-8 q^{-1 / 4} \quad \text { a.e. }
$$

Theorem 1.2 is a consequence of these equations, together with Lemma 3.3 . 


\section{REFERENCES}

[1] C. Aistleitner and I. Berkes. On the central limit theorem for $f\left(n_{k} x\right)$. Probab. Theory Relat. Fields, 146 (2010), no. 1-2, 267-289. MR2550364

[2] R. C. Baker. Metric number theory and the large sieve. J. London. Math. Soc. 24, 34-40, 1981. MR623668 (83a:10086)

[3] I. Berkes. On the asymptotic behaviour of $\sum f\left(n_{k} x\right)$. I. Main theorems, II. Applications. $Z$. Wahrscheinlichkeitstheorie und Verw. Gebiete 34:319-345; 347-365, 1976.

[4] I. Berkes. On the central limit theorem for lacunary trigonometric series. Anal. Math. 4:159180, 1978. MR514757 (80c:60032)

[5] I. Berkes and W. Philipp. An a.s. invariance principle for lacunary series $f\left(n_{k} x\right)$. Acta Math. Acad. Sci. Hungar. 34:141-155, 1979. MR546729 (80i:60042)

[6] I. Berkes and W. Philipp. The size of trigonometric and Walsh series and uniform distribution mod 1. J. London Math. Soc. (2), 50:454-464, 1994. MR.1299450 (96e:11099)

[7] J. W. S. Cassels. Some metrical theorems in Diophantine approximation III. Proc. Cambridge Philos. Soc. 46:219-225, 1950. MR0036789(12:162d)

[8] P. Erdős and J. F. Koksma. On the uniform distribution modulo 1 of sequences $(f(n, \theta))$. Proc. Kon. Nederl. Akad. Wetensch. 52:851-854, 1949. MR0032690 (11:331f)

[9] K. Fukuyama. The law of the iterated logarithm for discrepancies of $\left\{\theta^{n} x\right\}$. Acta Math. Hungar. 118:155-170, 2008. MR2378547 (2008m:60049)

[10] V. F. Gaposhkin. Lacunary series and independent functions (in Russian). Uspehi Mat. Nauk 21/6:3-82,1966. MR0206556 (34:6374)

[11] V. F. Gaposhkin. On the central limit theorem for some weakly dependent sequences (in Russian). Teor. Verojatn. Prim. 15:666-684, 1970. MR0282394(43:8106)

[12] M. Kac. On the distribution of values of sums of the type $\sum f\left(2^{k} t\right)$. Ann. of Math. (2) 47:33-49, 1946. MR0015548 (7:436f)

[13] M. Kac. Probability methods in some problems of analysis and number theory. Bull. Amer. Math. Soc. 55:641-665, 1949. MR.0031504(11:161b)

[14] H. Kesten. The discrepancy of random sequences $\{k x\}$, Acta Arith. 10:183-213, 1964/65. MR0168546 (29:5807)

[15] L. Kuipers and H. Niederreiter. Uniform Distribution of Sequences. Wiley, New York, 1974. MR0419394(54:7415)

[16] W. Philipp. Limit theorems for lacunary series and uniform distribution mod 1. Acta Arith. 26:241-251, 1975. MR0379420(52:325)

[17] R. Shorack and J. Wellner. Empirical processes with applications to statistics. Wiley, New York, 1986. MR838963(88e:60002)

[18] V. Strassen. Almost sure behavior of sums of independent random variables and martingales. Proc. Fifth Berkeley Sympos. Math. Statist. and Probability (Berkeley, Calif., 1965/66) Vol. II: Contributions to Probability Theory:315-343, 1967. MR0214118 (35:4969)

[19] S. Takahashi. An asymptotic property of a gap sequence. Proc. Japan Acad. 38:101-104, 1962. MR0140865 (25:4279)

[20] H. Weyl. Uber die Gleichverteilung von Zahlen mod. Eins. Math. Ann. 77:313-352, 1916. MR.1511862

[21] A. Zygmund. Trigonometric series. Vols. I, II. Third edition. Cambridge Mathematical Library. Cambridge University Press, Cambridge, 2002. MR1963498(2004h:01041)

Institute of Mathematics A, Graz University of Technology, Steyrergasse 30, 8010 Graz, Austria

E-mail address: aistleitner@finanz.math.tugraz.at 